\title{
The protective effect of supplemental calcium on colonic permeability depends on a calcium phosphate-induced increase in luminal buffering capacity
}

\author{
Marloes A. A. Schepens ${ }^{1,2}$, Sandra J. M. ten Bruggencate ${ }^{2}$, Arjan J. Schonewille ${ }^{1,2}$, \\ Robert-Jan M. Brummer ${ }^{3}$, Roelof van der Meer ${ }^{1,4}$ and Ingeborg M. J. Bovee-Oudenhoven ${ }^{1,2 *}$ \\ ${ }^{1}$ TI Food and Nutrition, PO Box 557, 6700 AN Wageningen, The Netherlands \\ ${ }^{2}$ NIZO Food Research, Department of Health, PO Box 20, 6710 BA Ede, The Netherlands \\ ${ }^{3}$ School of Health and Medical Sciences, Örebro University, Örebro, Sweden \\ ${ }^{4}$ Department of Human Nutrition, Wageningen University and Research Centre, Bomenweg 2, 6703 HD Wageningen, \\ The Netherlands
}

(Submitted 13 January 2011 - Final revision received 10 June 2011 - Accepted 20 June 2011 - First published online 19 August 2011)

\begin{abstract}
An increased intestinal permeability is associated with several diseases. Previously, we have shown that dietary Ca decreases colonic permeability in rats. This might be explained by a calcium-phosphate-induced increase in luminal buffering capacity, which protects against an acidic $\mathrm{pH}$ due to microbial fermentation. Therefore, we investigated whether dietary phosphate is a co-player in the effect of $\mathrm{Ca}$ on permeability. Rats were fed a humanised low-Ca diet, or a similar diet supplemented with Ca and containing either high, medium or low phosphate concentrations. Chromium-EDTA was added as an inert dietary intestinal permeability marker. After dietary adaptation, short-chain fructo-oligosaccharides (scFOS) were added to all diets to stimulate fermentation, acidify the colonic contents and induce an increase in permeability. Dietary Ca prevented the scFOS-induced increase in intestinal permeability in rats fed mediumand high-phosphate diets but not in those fed the low-phosphate diet. This was associated with higher faecal water cytotoxicity and higher caecal lactate levels in the latter group. Moreover, food intake and body weight during scFOS supplementation were adversely affected by the low-phosphate diet. Importantly, luminal buffering capacity was higher in rats fed the medium- and high-phosphate diets compared with those fed the low-phosphate diet. The protective effect of dietary Ca on intestinal permeability is impaired if dietary phosphate is low. This is associated with a calcium phosphate-induced increase in luminal buffering capacity. Dragging phosphate into the colon and thereby increasing the colonic phosphate concentration is at least part of the mechanism behind the protective effect of Ca on intestinal permeability.
\end{abstract}

Key words: Calcium: Phosphate: Short-chain fructo-oligosaccharides: Intestinal permeability

The intestinal tract is lined by a monolayer of epithelial cells, which is the largest and most important barrier between the body's internal milieu and the hostile external environment. Tight contact between the epithelial cells prevents access of luminal digestive enzymes, toxins, antigens and enteric microbiota to underlying tissue compartments ${ }^{(1-3)}$. Intestinal barrier dysfunction is suggested to be associated with the pathogenesis of a variety of intestinal diseases, including inflammatory bowel disease, coeliac disease, post-infectious irritable bowel syndrome and food allergy ${ }^{(1-5)}$.

Interestingly, dietary components can influence the epithelial barrier, in particular intestinal permeability, and hence possibly modulate disease development. We are interested in the effect of dietary $\mathrm{Ca}$, since it has been shown in several controlled studies that $\mathrm{Ca}$ is important for protection against intestinal infections with food-borne bacterial pathogens, both in rats and in human subjects ${ }^{(6-9)}$. In addition, Ca displayed cytoprotective effects in several studies in the field of colon carcinogenesis by precipitating cytotoxic surfactants, such as secondary bile acids ${ }^{(10,11)}$. Furthermore, supplemental $\mathrm{Ca}$ attenuated the development of colitis in HLA-B27 transgenic rats, which was associated with the prevention of a colitis-related increase in intestinal permeability due to $\mathrm{Ca}^{(12)}$. In a previous study ${ }^{(13)}$, we identified that the effect of dietary $\mathrm{Ca}$ on intestinal permeability is located in the colon. At present, however, it is still not clear how Ca exerts its

Abbreviations: CrEDTA, chromium-EDTA; HCaHP, high-calcium, high-phosphate; HCaLP, high-calcium, low-phosphate; HCaMP, high-calcium, mediumphosphate; LCaMP, low-calcium, medium-phosphate; scFOS, short-chain fructo-oligosaccharides.

*Corresponding author: I. M. J. Bovee-Oudenhoven, fax +31 318650 400, email ingeborg.bovee@nizo.nl 
protective effect on colonic permeability. Upon dietary intake, an insoluble calcium phosphate complex is formed in the small intestine, both in rats ${ }^{(14)}$ and in humans ${ }^{(15)}$. In this way, Ca prevents phosphate from being absorbed in the small intestine, and drags phosphate into the colon. In the colon, solubilisation of this complex increases the buffering capacity of the luminal contents, which protects the intestinal mucosa from being injured by an acidic $\mathrm{pH}$ due to microbial fermentation ${ }^{(16)}$. This mechanism might play a role in the effect of $\mathrm{Ca}$ on intestinal permeability, and it implies that dietary phosphate intake might be of importance for the effect of Ca on permeability.

In the present study, we investigated the potential effect of dietary phosphate on Ca-induced lowering of intestinal permeability, by applying high, medium or low phosphate concentrations in a high-Ca diet. Inert chromium-EDTA (CrEDTA) was added to the diets as an established marker for intestinal permeability ${ }^{(17,18)}$. After dietary adaptation for $10 \mathrm{~d}$, short-chain fructo-oligosaccharides (scFOS) were introduced in the diets. The intestinal fermentation of scFOS leads to a luminal organic acid load, which challenges the buffering capacity. We hypothesise that intestinal permeability will increase on a high-Ca diet with concomitant low dietary phosphate levels because of a decreased buffering capacity.

\section{Materials and methods}

\section{Experimental design: animals and diets}

The experimental protocol was approved by the animal welfare committee of Wageningen University and Research Centre (Wageningen, The Netherlands). Specific pathogenfree outbred male Wistar rats (WU; Harlan, Horst, The Netherlands), 8 weeks old and with a mean body weight of $288 \mathrm{~g}$ at the start of the experiment, were housed individually in metabolic cages. Animals were kept in a temperature- and humidity-controlled environment in a $12 \mathrm{~h}$ light $-12 \mathrm{~h}$ dark cycle. Rats (ten animals per dietary group) were fed a purified 'humanised' Western diet in restricted quantities $(16 \mathrm{~g} / \mathrm{d})$. Restricted feeding was necessary to prevent scFOS-induced differences in food consumption as observed earlier ${ }^{(19)}$ and hence differences in vitamin, mineral and CrEDTA intake. Demineralised drinking-water was supplied ad libitum. The reference diet (low-Ca, medium-phosphate; LCaMP), which has been used as a reference diet in our previous experiments $^{(12,13)}$, contained (per kg): $200 \mathrm{~g}$ acid casein, $326 \mathrm{~g}$ maize starch, $172 \mathrm{~g}$ glucose, $160 \mathrm{~g}$ palm oil, $40 \mathrm{~g}$ maize oil, $50 \mathrm{~g}$ cellulose, $2 \mathrm{~g}$ CrEDTA (see below) and $5.16 \mathrm{~g} \mathrm{CaHPO}_{4} \cdot 2 \mathrm{H}_{2} \mathrm{O}$ (corresponding to $30 \mathrm{mmol} \mathrm{Ca} / \mathrm{kg}$ diet; Sigma-Aldrich, St Louis, MO, USA). Vitamins and minerals (other than $\mathrm{Ca}$ ) were added to the diets according to AIN-93 ${ }^{(20)}$. The concentration of vitamins and minerals was increased by $20 \%$ to ensure adequate intake during restricted feeding. The diets had a high fat content to mimic the composition of a Western human diet. Dietary phosphate mainly originates from calcium phosphate and the protein source of the diet (casein: about $40 \mathrm{mmol}$ phosphate $/ \mathrm{kg}$ diet). The experimental diets were supplemented with $90 \mathrm{mmol} \mathrm{CaHPO}_{4} \cdot 2 \mathrm{H}_{2} \mathrm{O} / \mathrm{kg}$ diet (high-Ca, high-phosphate; HCaHP), or with $90 \mathrm{mmol} \mathrm{CaCl}_{2} \cdot 2 \mathrm{H}_{2} \mathrm{O} / \mathrm{kg}$ diet (high-Ca, medium-phosphate; HCaMP), at the expense of glucose. The high-Ca, low-phosphate diet (HCaLP) was supplemented with $90 \mathrm{mmol} \mathrm{CaCl}_{2} \cdot 2 \mathrm{H}_{2} \mathrm{O} / \mathrm{kg}$ diet, and the casein of this diet was replaced by whey protein isolate as a low-phosphate protein source (BiPRO, about $5 \mathrm{mmol}$ phosphate/kg diet; Davisco Foods International, Inc., Eden Prairie, MN, USA). Thus, the experiment consisted of four different diets: LCaMP diet (reference diet; $30 \mathrm{mmol} \mathrm{Ca}$ and $70 \mathrm{mmol}$ phosphate/kg diet), HCaHP diet (positive control since this diet has been used in our previous experiments, showing the effects of $\mathrm{Ca}$ on intestinal permeability ${ }^{(12,13)} ; 120 \mathrm{mmol}$ $\mathrm{Ca}$ and $160 \mathrm{mmol}$ phosphate/kg diet), HCaMP diet (120 mmol Ca and $70 \mathrm{mmol}$ phosphate/kg diet) and HCaLP diet $(120 \mathrm{mmol} \mathrm{Ca}$ and $35 \mathrm{mmol}$ phosphate $/ \mathrm{kg}$ diet). Inert CrEDTA was added to all diets to quantify intestinal permeability ${ }^{(18)}$. CrEDTA solution was prepared as described elsewhere and subsequently freeze-dried ${ }^{(21)}$. To check the complete formation and stability of the CrEDTA complex, the prepared CrEDTA solution was passed through a cationexchange resin column (Chelex 100 Resin; Bio-Rad, Hercules, CA, USA). No uncomplexed $\mathrm{Cr}^{3+}$ ions were present. Rats were fed the experimental diets for $10 \mathrm{~d}$, after which all diets were supplemented with $60 \mathrm{~g} \mathrm{scFOS} / \mathrm{kg}$ diet $\left(6 \%\right.$, w/w; Raftilose ${ }^{\circledR}$ P95; Orafti, Tienen, Belgium) at the expense of glucose. Food intake was recorded daily and animal weight twice every week. At experimental day 20, rats were anaesthetised with isoflurane and killed. The caecum was excised and caecal contents were collected.

\section{Measurement of intestinal permeability}

Total $24 \mathrm{~h}$ urine samples were collected at experimental days 10 and 20. For the CrEDTA measurement, urine was acidified with $50 \mathrm{~g} \mathrm{TCA} / 1$, centrifuged for $2 \mathrm{~min}$ at $14000 \mathrm{~g}$ and the supernatant was subsequently diluted with $0.5 \mathrm{~g} \mathrm{CsCl} / 1$. Then, chromium was analysed by inductively coupled plasma-atomic emission spectrophotometry.

\section{Analyses of faeces and caecal contents}

All faeces were collected during the last $3 \mathrm{~d}$ of experimental feeding without scFOS (pre-scFOS; experimental days 8, 9 and 10), and during the last $3 \mathrm{~d}$ of the scFOS supplementation period (experimental days 18, 19 and 20). Faeces and caecal contents were freeze-dried and subsequently ground to obtain homogeneous powdered samples. $\mathrm{Ca}$ and $\mathrm{P}$ were quantified in the faeces of the pre-scFOS period. To this end, faeces were treated with $50 \mathrm{~g}$ TCA/l, centrifuged for $2 \mathrm{~min}$ at $14000 \mathrm{~g}$, diluted with $0.5 \mathrm{~g} \mathrm{CsCl} / 1$, and analysed by inductively coupled plasma-atomic emission spectrophotometry. Buffering capacity was also determined in the faeces of the pre-scFOS period. To this end, pools of the freeze-dried faeces ( $2 \mathrm{~g}$ ) were reconstituted with doubledistilled water to $15 \%$ dry weight. The quantities of hydrochloric acid required to decrease the $\mathrm{pH}$ to 5 in these samples were measured, as described earlier ${ }^{(16)}$. For the cytotoxicity assay, faecal water was prepared by reconstituting freeze-dried 
(a)

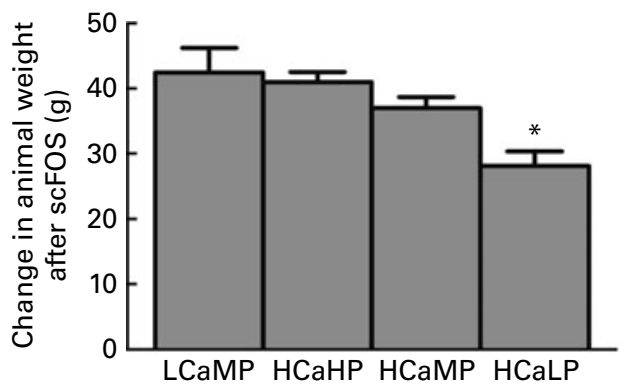

(b)

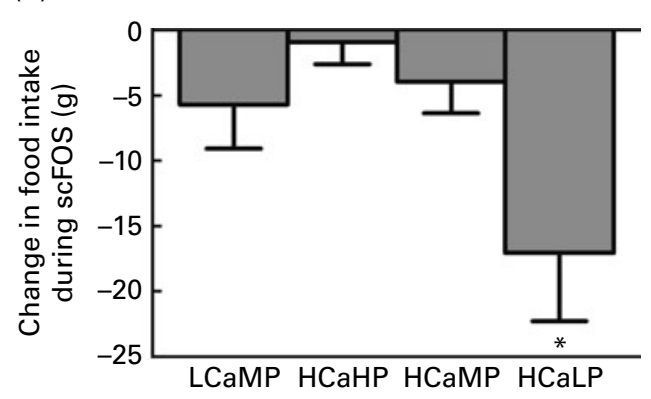

Fig. 1. Effect of dietary calcium and phosphate on (a) body-weight gain and (b) food intake during short-chain fructo-oligosaccharide (scFOS) supplementation ( $n$ 10). (a) Values are mean change in body weight at the end of the experiment (experimental day 20) compared with body weight just before the start of scFOS supplementation (experimental day 9 ), with standard errors represented by vertical bars. * Mean values were significantly different from those of rats on a highcalcium, medium-phosphate (HCaMP) diet $(P=0.006)$. (b) Values are mean cumulative change in food intake during scFOS supplementation compared with food consumption before scFOS supplementation, with standard errors represented by vertical bars. ${ }^{*}$ Mean values were significantly different from those of rats on a HCaMP diet $(P=0.02)$. LCaMP, low-calcium, medium-phosphate; HCaHP, high-calcium, high-phosphate; HCaLP, high-calcium, low-phosphate.

faeces with double-distilled water to $25 \%$ dry weight as described previously ${ }^{(22)}$. The cytotoxicity of the faecal water of the scFOS supplementation period was determined by potassium release of a human erythrocyte suspension after incubation with faecal water, as described earlier ${ }^{(23)}$, and validated earlier with intestinal epithelial cells ${ }^{(24)}$. Cytotoxicity was calculated and is expressed as a percentage of maximal lysis. Total lactic acid was determined in caecal contents using a colorimetric enzymatic kit (Enzyplus; BioControl Systems, Inc., Bellevue, WA, USA), as described earlier ${ }^{(25)}$.

\section{Statistical analysis}

All results are expressed as mean values with their standard errors. The predefined comparisons of interest were the LCaMP diet $v$. the HCaHP diet to study the effect of Ca (these two diets have been compared in our previous studies, showing the effects of $\mathrm{Ca}$ on intestinal permeability ${ }^{(12,13)}$ ), and the HCaHP diet $v$. the HCaMP diet and the HCaMP diet $v$. the HCaLP diet to study the effects of phosphate. Statistics were done by using one-way ANOVA or Kruskal-Wallis test, depending on the normality of the data. If differences were significant, this was followed by Student's $t$ test (for normally distributed data) or Mann-Whitney $U$ test (for non-normally distributed data) to identify the significant dietary effects. Differences were considered statistically significant when $P<0.05$ (all twosided). Statistical analyses were conducted with GraphPad Prism version 5.01 (GraphPad Software, Inc., La Jolla, CA, USA).

\section{Results}

\section{Animal growth and food intake}

No diet-induced differences in animal growth and food intake were observed before supplementation with scFOS: all rats consumed the provided $16 \mathrm{~g} / \mathrm{d}$ as intended. However, animal growth was affected by the different diets during scFOS supplementation. Rats from the HCaLP group gained less weight during supplementation with scFOS than those from the HCaMP group (Fig. 1(a), $P=0 \cdot 006$ ). The other dietary groups did not differ with respect to growth. The results for food intake were similar: HCaLP-fed rats had a more decreased food consumption than those fed the HCaMP diet during scFOS supplementation (Fig. 1(b), $P=0 \cdot 02$ ).

\section{Faecal baseline characteristics before short-chain fructo-oligosaccharide supplementation}

Daily output of faeces, based on dry weight, was increased due to Ca supplementation (Table $1 ; P<0 \cdot 0001$ ), in accordance with previous work ${ }^{(7,14)}$. To check whether the dietary interventions indeed affected the baseline $\mathrm{Ca}$ and phosphate levels in the colonic lumen before scFOS supplementation, we measured total $\mathrm{Ca}$ and $\mathrm{P}$ in the faeces (Table 1). Indeed, Ca concentration was clearly higher in the Ca-supplemented group (HCaHP: $P<0.0001$ ) compared with levels in the LCaMP group. $\mathrm{P}$ levels in the faeces also corresponded well with the dietary intervention. Faecal $\mathrm{P}$ was increased in rats

Table 1. Effect of diet on faecal baseline characteristics before short-chain fructo-oligosaccharide supplementation (Mean values with their standard errors)

\begin{tabular}{|c|c|c|c|c|c|c|c|c|}
\hline & \multicolumn{2}{|c|}{ LCaMP } & \multicolumn{2}{|c|}{$\mathrm{HCaHP}$} & \multicolumn{2}{|c|}{ HCaMP } & \multicolumn{2}{|c|}{ HCaLP } \\
\hline & Mean & SEM & Mean & SEM & Mean & SEM & Mean & SEM \\
\hline Faecal dry weight $(\mathrm{g} / \mathrm{d})$ & 1.02 & 0.02 & $1 \cdot 31^{*}$ & 0.02 & 1.37 & 0.03 & 1.38 & 0.04 \\
\hline Ca excretion $(\mu \mathrm{mol} / \mathrm{d})$ & 78 & 10 & $1051^{*}$ & 34 & 1098 & 19 & $887 \dagger$ & 19 \\
\hline P excretion $(\mu \mathrm{mol} / \mathrm{d})$ & 65 & 6 & $733^{*}$ & 21 & $350 \ddagger$ & 17 & $116 \dagger$ & 8 \\
\hline
\end{tabular}

LCaMP, low-ca, medium-phosphate; HCaHP, high-ca, high-phosphate; HCaMP, high-ca, medium-phosphate; HCaLP, high-ca, low-phosphate.

${ }^{*}$ Mean values were significantly different from those of rats on the LCaMP diet $(P<0.0001)$.

† Mean values were significantly different from those of rats on the HCaMP diet $(P<0.0001)$.

$\ddagger$ Mean values were significantly different from those of rats on the HCaHP diet $(P<0.0001)$. 


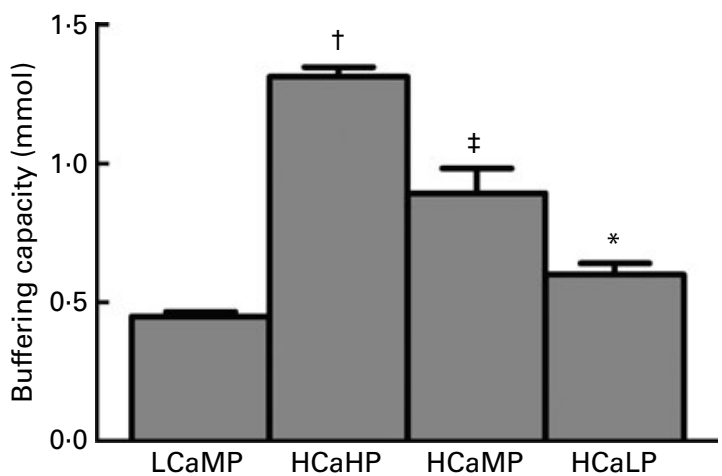

Fig. 2. Effect of dietary calcium and phosphate on faecal buffering capacity before short-chain fructo-oligosaccharide supplementation $(n 10)$. Values are means of triplicate measurements of pooled faeces (15\% dry weight), with standard errors represented by vertical bars. Buffering capacity is calculated as the quantities of hydrochloric acid (in mmol) required to decrease the $\mathrm{pH}$ to $5 .{ }^{*}$ Mean values were significantly different from those of rats on a highcalcium, medium-phosphate (HCaMP) diet $(P=0.04)$. † Mean values were significantly different from those of rats on a low-calcium, medium-phosphate (LCaMP) diet $(P<0.0001)$. $¥$ Mean values were significantly different from those of rats on a high-calcium, high-phosphate $(\mathrm{HCaHP})$ diet $(P=0.01)$. HCaLP, high-calcium, low-phosphate.

fed the HCaHP diet $(P<0.0001)$ compared with those fed the LCaMP diet. As expected, the concentration of $\mathrm{P}$ was decreased in the HCaMP group $(P<0.0001)$ compared with the HCaHP group, and further diminished in rats fed the HCaLP diet compared with the HCaMP group $(P<0 \cdot 0001)$. Subsequently, the buffering capacity of these faecal samples was determined to evaluate whether the diets affected this capacity as hypothesised. Luminal buffering capacity was higher in the HCaHP group $(P<0.0001)$ in comparison with rats fed the LCaMP diet (Fig. 2). Also as expected, buffering capacity of the faeces was lower in rats on the HCaMP diet $(P=0 \cdot 01)$ compared with those on the HCaHP diet. And indeed, buffering capacity was further decreased in rats fed the HCaLP diet $(P=0.04)$ compared with those fed the HCaMP diet. The results of the faecal buffering capacity measurements after scFOS supplementation showed similar dietary effects (data not shown).

The effect of dietary calcium and phosphate on intestinal permeability

The main outcome of the present study is the dietary effect on intestinal permeability, measured by urinary CrEDTA excretion. Dietary Ca already decreased intestinal permeability before the colon was challenged with scFOS; however, this was not the case in rats fed the HCaLP diet. Urinary CrEDTA expressed as a percentage of dietary intake was 5.9 (SEM $0 \cdot 5)$ in the LCaMP group, $4 \cdot 2$ (SEM $0 \cdot 3)$ in the HCaHP group ( $P=0.007$, compared with LCaMP), 4.1 (SEM 0.2) in the HCaMP group and 6.5 (SEm 0.5 ) in the HCaLP group ( $P=0.0002$, compared with HCaMP). Importantly, after scFOS supplementation, dietary Ca only prevented the fermentation-induced increase in intestinal permeability when rats were fed a diet containing high or medium levels of phosphate (HCaHP, $P=0.049$ compared with LCaMP; Fig. 3), but not when rats were fed the low-phosphate diet ( $P=0.04$ compared with HCaMP; Fig. 3).

\section{Dietary effects on caecal lactate levels}

To investigate whether fermentation of scFOS is disturbed due to acid accumulation, lactate was quantified in caecal contents $^{(26,27)}$. Ca supplementation in a high-phosphate background ( $\mathrm{HCaHP})$ resulted in significantly lower lactate levels in caecal contents compared with rats fed the LCaMP diet $(P=0.006$; Fig. 4(a)). Lactate levels were similar in the HCaMP group compared with rats fed the HCaHP diet, while these levels were higher in rats on the HCaLP diet compared with the HCaMP group $(P=0 \cdot 01$; Fig. 4(a)). Interestingly, lactate levels are correlated with the colonic permeability results (Spearman's $r 0 \cdot 45, P=0 \cdot 006$ ). The dietary effects on caecal $\mathrm{pH}$ show comparable results, since the $\mathrm{pH}$ in the caecum of the HCaHP group was significantly higher than the $\mathrm{pH}$ of the caecal contents of LCaMP-fed rats (6.9 (SEM 0.2) v. 5.8 (SEM 0.1), respectively, $P=0 \cdot 0008$ ). No differences in caecal $\mathrm{pH}$ were observed in the other dietary groups (HCaMP, $7 \cdot 2$ (sem 0.2); HCaLP, $6 \cdot 8$ (sem 0.3)).

\section{Dietary calcium and phosphate influence luminal cytotoxicity}

To investigate whether faecal water cytotoxicity, reflecting colonic mucosal exposure to luminal irritants, plays a role in the dietary effects on colonic permeability, the cytotoxicity assay was performed with faecal water from the period after scFOS supplementation. Cytotoxic activity of faecal water was lower in rats fed the HCaHP diet $(P=0.006$; Fig. 4(b)) compared with those fed on the LCaMP diet. In HCaLP-fed rats, luminal cytotoxicity was increased compared with rats fed the HCaMP diet $(P=0 \cdot 01$; Fig. 4(b)).

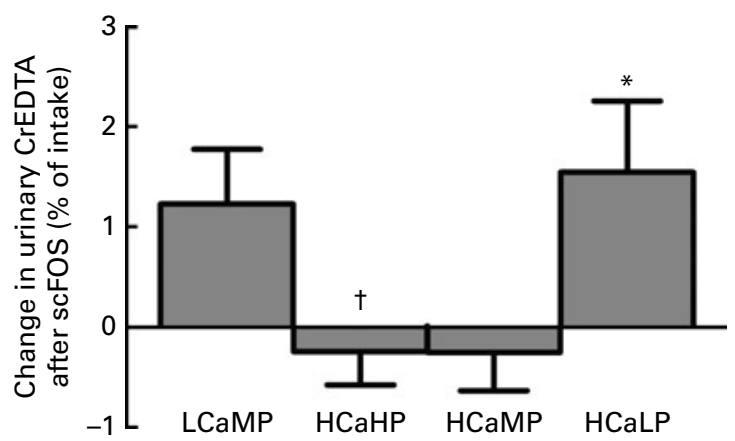

Fig. 3. Effect of dietary calcium and phosphate on urinary chromium-EDTA (CrEDTA) excretion, a marker for intestinal paracellular permeability, after short-chain fructo-oligosaccharide (scFOS) supplementation (n 10). Values are mean change in urinary CrEDTA excretion (\% of intake) at the end of the experiment (experimental day 20) compared with urinary CrEDTA excretion (\% of intake) before the start of scFOS supplementation (experimental day 10), with standard errors represented by vertical bars. ${ }^{*}$ Mean values were significantly different from those of rats on a high-calcium, mediumphosphate (HCaMP) diet $(P=0.04)$. † Mean values were significantly different from those of rats on a low-calcium, medium-phosphate (LCaMP) diet $(P=0.049)$. HCaHP, high-calcium, high-phosphate; HCaLP, high-calcium, low-phosphate. 
(a)

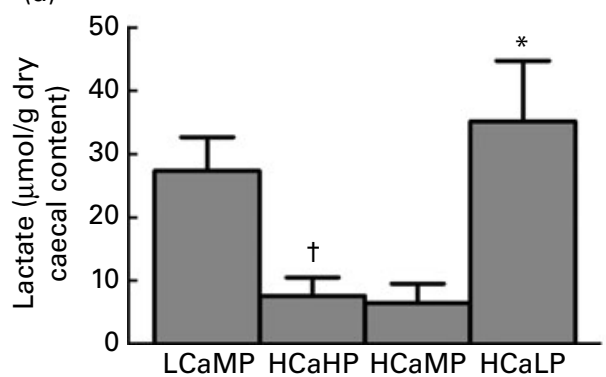

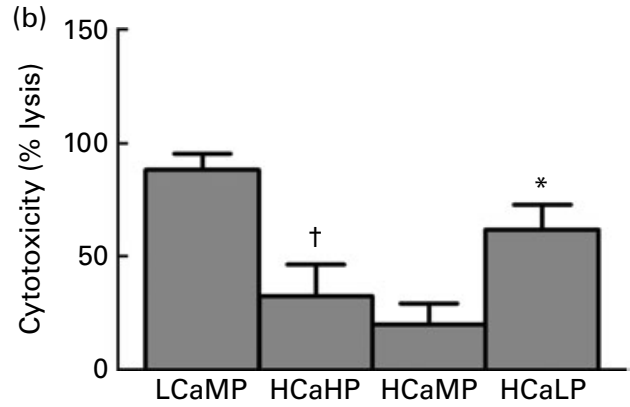

Fig. 4. Effect of dietary calcium and phosphate on (a) caecal lactate levels and (b) luminal cytotoxicity after short-chain fructo-oligosaccharide supplementation (n 10). Values are means of caecal lactate levels quantified by using a colorimetric enzymatic kit, and for luminal cytotoxicity determined with an erythrocyte bioassay, with standard errors represented by vertical bars. * Mean values were significantly different from those of rats on a high-calcium, medium-phosphate $(\mathrm{HCaMP})$ diet $(P=0.01)$. † Mean values were significantly different from those of rats on a low-calcium, medium-phosphate $(\mathrm{LCaMP})$ diet $(P=0.006)$. HCaHP, high-calcium, high-phosphate; HCaLP, high-calcium, low-phosphate.

\section{Discussion}

The present study shows that the beneficial effect of dietary Ca on colonic permeability is impaired if dietary phosphate levels are low. If dietary phosphate is sufficient, Ca decreases colonic permeability, which is associated with an increase in luminal buffering capacity. The protective effect of Ca on permeability is also related to lower caecal lactate levels and a decreased faecal cytotoxicity during scFOS supplementation. Besides the abrogation of the protective effect of a high-Ca diet on colonic permeability, low phosphate intake also adversely affected food intake and animal weight after scFOS supplementation.

The protective effect of dietary Ca on intestinal permeability has been shown consistently in several studies using different animal models. It is of relevance that Ca can improve intestinal permeability both in a healthy situation ${ }^{(13)}$ and during intestinal infection ${ }^{(9)}$ and colitis development ${ }^{(12)}$. In the latter two studies, the prevention of an increase in intestinal permeability by $\mathrm{Ca}$ was associated with an enhanced resistance to the development of intestinal disease. The present study emphasises the potential of $\mathrm{Ca}$ to decrease intestinal permeability in a healthy condition; however, it also shows that dietary phosphate intake has to be taken into account.

Since it is still unknown how Ca exerts its beneficial effect on gut permeability, we aimed to investigate whether a calcium-phosphate-induced increase in luminal buffering capacity is involved. By acidifying the colon with scFOS in the present study, the capacity to buffer the intestinal contents becomes even more important. Interestingly, scFOS induce an increase in intestinal permeability ${ }^{(13,28)}$, but it is not proven that this is associated with luminal acidification caused by scFOS, although this seems likely. There are indications from in vitro studies that the extracellular $\mathrm{pH}$ is important for paracellular permeability ${ }^{(29-31)}$. We aimed to show experimental support for the hypothesised effect that the luminal buffering capacity affects intestinal permeability, and is part of the protective effect of $\mathrm{Ca}$, in a situation of enhanced luminal acidification through scFOS in the diet. Indeed, the protective effect of $\mathrm{Ca}$ on colonic permeability was impaired if dietary phosphate levels were low, and this effect was associated with a decrease in luminal buffering capacity.
Fermentation already starts in the caecum of rats; however, as has been described earlier ${ }^{(13)}$, it clearly continues in the rat colon, showing the relevance of faecal sampling. Therefore, the similarity of the effects of scFOS in both the caecum and colon of rats and human colonic fermentation supports the use of the rat as an appropriate model to study possible subsequent permeability alterations. Moreover, intestinal $\mathrm{pH}$ values ${ }^{(32)}$ and faecal phosphate levels ${ }^{(33)}$ are similar in rats and humans, indicating that diet-modulated buffering capacity in the colon is comparable between these species. These results show that the protective effect of dietary Ca is at least partly due to its role as a carrier of phosphate into the colon. Subsequently, phosphate is responsible for the increase in colonic buffering capacity.

Ca supplementation in combination with high or medium dietary phosphate prevented a fructo-oligosaccharide-induced accumulation of lactate in the caecal contents, which was associated with the decrease in intestinal permeability. During rapid fermentation of easily fermentable carbohydrates, lactate accumulation can occur when micro-organisms that utilise lactate are inhibited ${ }^{(26)}$. These micro-organisms might be inhibited by an acidic $\mathrm{pH}$ due to a compromised buffering capacity. It is therefore likely that alterations in microbiota composition or activity are responsible for the changes in caecal lactate levels. Interestingly, intestinal micro-organisms, for example Escherichia coli, are able to induce an increase in intestinal permeability ${ }^{(34)}$. Therefore, buffering capacity might also indirectly influence intestinal permeability by modulating the gut microbiota.

An alternative explanation for the dietary effects on intestinal permeability, besides modulation of luminal buffering capacity, might be the influence of supplemental Ca on luminal cytotoxicity. The present study demonstrates that a lack of phosphate counteracts the inhibiting effect of Ca on cytotoxicity ${ }^{(35)}$. Clearly, both $\mathrm{Ca}$ and phosphate are needed to precipitate cytotoxic components ${ }^{(35)}$, which can irritate the intestinal epithelium ${ }^{(36)}$ and subsequently modify permeability. Modulation of luminal cytotoxicity might also change the microbiota, which can thereby exert an influence on epithelial integrity $^{(34)}$. We have shown earlier that $\mathrm{Ca}$ affects the gut microbiota $^{(9,13,37)}$. The results of the present study suggest that phosphate is necessary for the effect of $\mathrm{Ca}$, but not 
determinative, i.e. as long as phosphate intake is sufficient ( $\geq 70 \mathrm{mmol} / \mathrm{kg}$ diet), the effect of $\mathrm{Ca}$ is not dependent on phosphate.

Impairment of the gut mucosal barrier by scFOS has been shown earlier in association with a decreased resistance to intestinal infection ${ }^{(19,28,38)}$. These harmful effects were decreased when Ca was supplemented to the $\operatorname{diet}^{(8)}$. The present study emphasises again that it is important to take care of a sufficient $\mathrm{Ca}$ intake when non-digestible carbohydrates are consumed. Furthermore, the present results show that dietary phosphate levels should also be taken into consideration. The adverse effects of low phosphate intake on food consumption and animal weight, which emerged during scFOS supplementation, support this observation.

The $\mathrm{Ca}$ and phosphate content of the rat diets in the present study is nutritionally relevant for the human diet. In general, human dietary $\mathrm{Ca}$ intake in the Western world ranges from 600 to $1100 \mathrm{mg}$ daily ${ }^{(39)}$. The Ca concentration of the low-Ca diet corresponds to a daily $\mathrm{Ca}$ intake of $600 \mathrm{mg}$ in humans, while the Ca-supplemented diets provided more than the general habitual dietary $\mathrm{Ca}$ intake (comparable to $2 \cdot 4 \mathrm{~g}$ daily), which is not unrealistic when taking $\mathrm{Ca}$ supplements ${ }^{(12)}$. In addition, the animal diets contained phosphate levels of approximately $160 \mathrm{mmol} / \mathrm{kg}$ diet (high-phosphate), $70 \mathrm{mmol} /$ $\mathrm{kg}$ diet (medium-phosphate) and $35 \mathrm{mmol} / \mathrm{kg}$ diet (lowphosphate), which are in the range of human intake of about $40 \mathrm{mmol}$ phosphate daily ( $80 \mathrm{mmol} / \mathrm{kg}$ diet), assuming that humans have a daily dry food intake of about $500 \mathrm{~g}^{(39)}$.

In conclusion, the present study shows that the protective effect of dietary $\mathrm{Ca}$ on intestinal permeability is impaired if phosphate levels are low, and this is associated with the effects on luminal buffering capacity. This is particularly important when consuming a diet with non-digestible and rapidly fermentable carbohydrates such as scFOS. So, phosphate levels should be sufficient in the diet to accomplish the protective effect of $\mathrm{Ca}$ on intestinal permeability. It cannot be excluded that changes in luminal buffering capacity also indirectly modulate intestinal permeability, for example, by the effects on the intestinal microbiota. The present study encourages follow-up studies, particularly in human subjects, to further explore and apply the potential to modulate gut barrier integrity by relatively simple dietary interventions.

\section{Acknowledgements}

The authors wish to thank the biotechnicians at the Small Animal Centre (Wageningen University and Research Centre, The Netherlands) for expert assistance. This study was supported by TI Food and Nutrition (Wageningen, The Netherlands). None of the authors had conflicts of interest. M. A. A. S. contributed to the study design, experimental procedures, data analysis, data interpretation and manuscript writing. S. J. M. t. B contributed to the study design, data interpretation and manuscript writing. A. J. S. assisted in the experimental procedures and data analysis. R.-J. M. B. contributed to the data interpretation and assisted in manuscript writing. R. V. d. M. and I. M. J. B.-O. contributed to the study design, data interpretation and manuscript writing. All authors read and approved the final submitted manuscript.

\section{References}

1. Arrieta MC, Bistritz L \& Meddings JB (2006) Alterations in intestinal permeability. Gut 55, 1512-1520.

2. Groschwitz KR \& Hogan SP (2009) Intestinal barrier function: molecular regulation and disease pathogenesis. J Allergy Clin Immunol 124, 3-20; quiz 21-22.

3. Turner JR (2009) Intestinal mucosal barrier function in health and disease. Nat Rev Immunol 9, 799-809.

4. Farhadi A, Banan A, Fields J, et al. (2003) Intestinal barrier: an interface between health and disease. J Gastroenterol Hepatol 18, 479-497.

5. Spiller RC (2007) Role of infection in irritable bowel syndrome. J Gastroenterol 42, Suppl. 17, 41-47.

6. Bovee-Oudenhoven IM, Lettink-Wissink ML, Van Doesburg W, et al. (2003) Diarrhea caused by enterotoxigenic Escherichia coli infection of humans is inhibited by dietary calcium. Gastroenterology 125, 469-476.

7. Bovee-Oudenhoven IM, Termont DS, Weerkamp AH, et al. (1997) Dietary calcium inhibits the intestinal colonization and translocation of Salmonella in rats. Gastroenterology 113, 550-557.

8. Ten Bruggencate SJ, Bovee-Oudenhoven IM, Lettink-Wissink ML, et al. (2004) Dietary fructo-oligosaccharides and inulin decrease resistance of rats to salmonella: protective role of calcium. Gut 53, 530-535.

9. Ten Bruggencate SJ, Snel J, Schoterman MH, et al. (2011) Efficacy of various dietary calcium salts to improve intestinal resistance to Salmonella infection in rats. Br J Nutr $\mathbf{1 0 5}$, 489-495.

10. Govers MJ, Termont DS, Lapre JA, et al. (1996) Calcium in milk products precipitates intestinal fatty acids and secondary bile acids and thus inhibits colonic cytotoxicity in humans. Cancer Res 56, 3270-3275.

11. Lapre JA, De Vries HT, Koeman JH, et al. (1993) The antiproliferative effect of dietary calcium on colonic epithelium is mediated by luminal surfactants and dependent on the type of dietary fat. Cancer Res 53, 784-789.

12. Schepens MA, Schonewille AJ, Vink C, et al. (2009) Supplemental calcium attenuates the colitis-related increase in diarrhea, intestinal permeability, and extracellular matrix breakdown in HLA-B27 transgenic rats. $J$ Nutr 139, 1525-1533.

13. Schepens MA, Rijnierse A, Schonewille AJ, et al. (2010) Dietary calcium decreases but short-chain fructo-oligosaccharides increase colonic permeability in rats. $\mathrm{Br} J$ Nutr $\mathbf{1 0 4}$, $1780-1786$.

14. Govers MJ \& Van der Meer R (1993) Effects of dietary calcium and phosphate on the intestinal interactions between calcium, phosphate, fatty acids, and bile acids. Gut $\mathbf{3 4}$, $365-370$

15. Van der Meer R, Welberg JW, Kuipers F, et al. (1990) Effects of supplemental dietary calcium on the intestinal association of calcium, phosphate, and bile acids. Gastroenterology 99, $1653-1659$.

16. Remesy C, Levrat MA, Gamet L, et al. (1993) Cecal fermentations in rats fed oligosaccharides (inulin) are modulated by dietary calcium level. Am J Physiol 264, G855-G862.

17. Aabakken L (1989) Cr-ethylenediaminetetraacetic acid absorption test. Methodologic aspects. Scand J Gastroenterol 24, 351-358. 
18. Arslan G, Atasever T, Cindoruk M, et al. (2001) (51)CrEDTA colonic permeability and therapy response in patients with ulcerative colitis. Nucl Med Commun 22, 997-1001.

19. Bovee-Oudenhoven IM, ten Bruggencate SJ, Lettink-Wissink ML, et al. (2003) Dietary fructo-oligosaccharides and lactulose inhibit intestinal colonisation but stimulate translocation of salmonella in rats. Gut 52, 1572-1578.

20. Reeves PG, Nielsen FH \& Fahey GC Jr (1993) AIN-93 purified diets for laboratory rodents: final report of the American Institute of Nutrition ad hoc writing committee on the reformulation of the AIN-76A rodent diet. J Nutr 123, 1939-1951.

21. Binnerts W, Van het Klooster A \& Frens A (1968) Soluble chromium indicator measured by atomic absorption in digestion experiments. Vet Rec 82, 470 .

22. Sesink AL, Termont DS, Kleibeuker JH, et al. (1999) Red meat and colon cancer: the cytotoxic and hyperproliferative effects of dietary heme. Cancer Res 59, 5704-5709.

23. Bovee-Oudenhoven I, Termont D, Dekker R, et al. (1996) Calcium in milk and fermentation by yoghurt bacteria increase the resistance of rats to Salmonella infection. Gut 38, 59-65.

24. Lapre JA, Termont DS, Groen AK, et al. (1992) Lytic effects of mixed micelles of fatty acids and bile acids. Am J Physiol 263, G333-G337.

25. Bovee-Oudenhoven IM, Termont DS, Heidt PJ, et al. (1997) Increasing the intestinal resistance of rats to the invasive pathogen Salmonella enteritidis: additive effects of dietary lactulose and calcium. Gut 40, 497-504.

26. Cummings JH (1981) Short chain fatty acids in the human colon. Gut 22, 763-779.

27. Hashizume K, Tsukahara T, Yamada K, et al. (2003) Megasphaera elsdenii JCM1772T normalizes hyperlactate production in the large intestine of fructooligosaccharidefed rats by stimulating butyrate production. J Nutr $\mathbf{1 3 3}$, 3187-3190.

28. Ten Bruggencate SJ, Bovee-Oudenhoven IM, Lettink-Wissink ML, et al. (2005) Dietary fructooligosaccharides increase intestinal permeability in rats. $J$ Nutr 135, 837-842.

29. Lim TS, Vedula SR, Hui S, et al. (2008) Probing effects of $\mathrm{pH}$ change on dynamic response of claudin-2 mediated adhesion using single molecule force spectroscopy. Exp Cell Res 314, 2643-2651.

30. Menconi MJ, Salzman AL, Unno N, et al. (1997) Acidosis induces hyperpermeability in $\mathrm{Caco}-2 \mathrm{BBe}$ cultured intestinal epithelial monolayers. Am J Physiol 272, G1007-G1021.

31. Unno N, Menconi MJ, Smith M, et al. (1997) Hyperpermeability of intestinal epithelial monolayers is induced by NO: effect of low extracellular pH. Am J Physiol 272, G923-G934.

32. Kararli TT (1995) Comparison of the gastrointestinal anatomy, physiology, and biochemistry of humans and commonly used laboratory animals. Biopharm Drug Dispos 16, 351-380.

33. Ten Bruggencate SJ, Bovee-Oudenhoven IM, Lettink-Wissink ML, et al. (2006) Dietary fructooligosaccharides affect intestinal barrier function in healthy men. $J$ Nutr 136, 70-74.

34. Mangell P, Nejdfors P, Wang M, et al. (2002) Lactobacillus plantarum 299v inhibits Escherichia coli-induced intestinal permeability. Dig Dis Sci 47, 511-516.

35. Van der Meer R, Termont DS \& De Vries HT (1991) Differential effects of calcium ions and calcium phosphate on cytotoxicity of bile acids. Am J Physiol 260, G142-G147.

36. Saunders DR, Hedges JR, Sillery J, et al. (1975) Morphological and functional effects of bile salts on rat colon. Gastroenterology 68, 1236-1245.

37. Bovee-Oudenhoven IM, Wissink ML, Wouters JT, et al. (1999) Dietary calcium phosphate stimulates intestinal lactobacilli and decreases the severity of a salmonella infection in rats. J Nutr 129, 607-612.

38. Ten Bruggencate SJ, Bovee-Oudenhoven IM, Lettink-Wissink ML, et al. (2003) Dietary fructo-oligosaccharides dosedependently increase translocation of salmonella in rats. $J$ Nutr 133, 2313-2318.

39. Alaimo K, McDowell MA, Briefel RR, et al. (1994) Dietary intake of vitamins, minerals, and fiber of persons ages 2 months and over in the United States: Third National Health and Nutrition Examination Survey, Phase 1, 1988-91. Adv Data 1-28. 